\title{
Volumetric modulated arc therapy (VMAT) is superior to intensity modulated radiotherapy (IMRT) for liver sparing in SBRT for hepatocellular carcinoma
}

Yulia Kundel ( $\nabla$ yuliak@clalit.org.il )

Rabin Medical Center

Yasmin Korzets

Rabin Medical Center

Miriam Weinstock-Sabbah

Rabin Medical Center

Assaf Moore

Rabin Medical Center

Noa Gordon

Rabin Medical Center

Salomon M. Stemmer

Rabin Medical Center

Aaron Popovtzer

Rabin Medical Center

Baruch Brenner

Rabin Medical Center

Eyal Fenig

Rabin Medical Center

Aaron M. Allen

Rabin Medical Center

Research

Keywords: VMAT, hepatocellular carcinoma, SBRT

Posted Date: October 2nd, 2020

DOl: https://doi.org/10.21203/rs.3.rs-84167/v1

License: (9) This work is licensed under a Creative Commons Attribution 4.0 International License.

Read Full License 
Page 2/11 


\section{Abstract}

Background: The best delivery modality of stereotactic body radiotherapy (SBRT) for hepatocellular carcinoma (HCC) from a technical point of view is still a matter of debate. The purpose of this study was to compare planning parameters with volumetric modulated arc therapy (VMAT) to static intensity modulated radiotherapy (IMRT) in treatment of HCC treated with SBRT.

Methods: Twenty patients (pts) with localized HCC who were treated with SBRT were re-planned using two different radiation techniques: IMRT and VMAT. Patients with Child A cirrhosis received 45-54 Gy in 3 fractions and 5 pts with Child B cirrhosis received $30 \mathrm{~Gy}$ in 5 fractions. Planning was optimized to minimize doses to organs at risk (OAR) without compromising coverage of the planning treatment volume (PTV). VMAT and IMRT plans were compared using the conformity index (Cl), homogeneity index of the PTV and monitor units (MUs) for time of treatment delivery, and other dose volume histogram (DVH) metrics.

Results: The $\mathrm{Cl}$ of VMAT plans were superior to those of IMRT $(1.11 \pm 0.05$ vs $1.18 \pm 0.06(p<0.05)$ The MUs were significantly lower for VMAT $(423.78 \pm 50.65)$ than for IMRT $(890 \pm 160.68)(p<0.01)$. Stomach max dose, normal liver V15 Gy, normal liver mean dose were also decreased with VMAT planning. This was achieved without increased :V30 Gy of duodenum and small bowel , $10 \mathrm{cc}(\mathrm{Gy})$ of duodenum, mean dose of right kidney and cord max dose .

Conclusion: PTV coverage was more conformal with VMAT planning, with lower MUs and shorter delivery time compared to IMRT in all pts. Moreover VMAT planning was more effective than IMRT planning in the sparing of normal liver and stomach.

\section{Background}

Hepatocellular carcinoma represents the sixth most commonly occurring cancer in the world and the third largest cause of cancer mortality. Treatment options include liver transplantation, surgical resection, radiofrequency ablation, percutaneous ethanol injection, transarterial embolization/chemoembolization, sorafenib and radiation therapy. In the past, radiation induced liver disease (RILD) has greatly limited the role of radiation due to the relationship between irradiated liver volume and toxicity [1].

Developments of radiotherapy techniques, mainly IMRT, have gradually made radiotherapy more relevant in the treatment of HCC and indications for its use have expanded in the last few years. SBRT has been shown as an alternative option in early stage HCC patients, patients not eligible for other ablative procedures or as a bridge to liver transplant.

SBRT delivers high doses of radiation in a few fractions with high degree of accuracy and has shown encouraging results. [2]. Studies comparing IMRT and VMAT to 3DCRT SBRT in patients with lung cancer, pancreatic cancer, spinal and abdominal metastases showed improvements in dose conformity and homogeneity with VMAT $[3,4]$. 
The best delivery modality for HCC SBRT from a technical point of view is a matter of debate. We compared two IMRT techniques: (1) Sliding window IMRT, in which modulated multileaf collimator in multiple static radiation fields is used and (2) VMAT, which delivers an entire IMRT treatment in a single gantry rotation around the patient. Current literature comparing VMAT and IMRT for lung tumors shows that both techniques could provide comparable target coverage and dose conformity however, OAR results do not show a clear preference. The purpose of this study was to better ascertain whether one technique is preferable to the other in terms of coverage, conformality, homogeneity and dose to OAR in SBRT for HCC.

\section{Methods}

\section{Patient population}

Twenty patients (pts) with single lesion HCC who were treated with SBRT at our center were entered into this planning study, after obtaining Helsinki committee approval. Median age at RT was 62.2 (range, 43.083.9) years, $70 \%$ were male, $70 \%$ were viral carriers, and all had liver cirrhosis. Fifteen pts had Child-Pugh A cirrhosis and 5 pts had Child-Pugh B cirrhosis. ECOG performance status was $\leq 2$. All patients had early stage disease (BCLC stage A). Five patients had left lobe tumors and 15 patients had right lobe tumors.

\section{Immobilization, simulation, and target delineation}

The patients were immobilized using vacuum casts in a supine position with arms raised above their heads. Contrast multiphase 4-dimentional CT simulation was performed with a 2.5-mm slice thickness and included whole liver and bilateral kidneys. Respiratory control and abdominal compression were used. Images were reconstructed on the Advantage Workstation (GE Healthcare, Chicago, IL). The internal target volumes were created using all 10 phases to account for maximal tumor excursion.

The gross tumor volume (GTV) was defined as the contrast enhancing tumor volume on a triphasic CT or MRI scan. The PTV was defined as a 2-3 mm margin around the GTV. The PTV was reduced in case of proximity to vital normal tissue.

Organs at risk were also delineated, including whole liver, non-target liver (whole liver minus PTV), stomach, duodenum, kidney, spinal cord and small bowel. Before each treatment, cone beam CT was used to position the patient.

\section{Treatment planning and dose delivery}

In our study, we prescribed $95 \%$ of total dose to cover $\geq 95 \%$ of the PTV coverage while keeping the minimum dose $\geq 93 \%$ of total dose and maximum dose $\leq 107 \%$ of total dose and normalized all plans to the mean dose of PTV.

Fifteen patients with Child A cirrhosis received 45-54 Gy in 3 fractions and five patients with Child B cirrhosis received $30 \mathrm{~Gy}$ in 5 fractions. Patients were treated every other day. For the purposes of the 
planning study, each patient case was replanned with both IMRT and VMAT techniques. The plans were calculated using the ECLIPSE planning system (Varian medical systems, Palo Alto, CA).

The VMAT plans were optimized using 1 to 3 arcs with a total arc span varying between 190 to 490 degrees. For IMRT, (6-8 fixed fields were used and) almost all plans were optimized with 7 fixed fields. Maximum dose rate was used for all plans: $600 \mathrm{MU} / \mathrm{min}$ for $6 \mathrm{MV}$ and $10 \mathrm{MV}$ plans and $1400 \mathrm{MU} / \mathrm{min}$ and $2400 \mathrm{MU} / \mathrm{min}$ for $6 \mathrm{MV}-\mathrm{FFF}$ and $10 \mathrm{MV}$-FFF plans, respectively.

For patients with Child A cirrhosis, one third of the uninvolved liver was restricted to $10 \mathrm{~Gy}$, and $500 \mathrm{cc}$ of uninvolved liver received $<7 \mathrm{~Gy}$. For patients with Child B cirrhosis, one third of the uninvolved liver was restricted to $18 \mathrm{~Gy}$, and $500 \mathrm{cc}$ of uninvolved liver received < $12 \mathrm{~Gy}$. The complete RT constraints can be viewed in Table 1

Table 1

DVH constraints for organs at risk

\begin{tabular}{|ll|}
\hline Organ & Constraints \\
\hline Uninvolved liver & $\begin{array}{l}\mathrm{V} 5<50 \%, \mathrm{~V} 7<30 \%, \mathrm{~V} 15<700 \mathrm{cc} \\
\end{array}$ \\
\hline Ft. kidney & $\mathrm{V} 15<35 \%$ \\
\hline Small bowel/duodenum/stomach & $\max$ dose $<30 \mathrm{~Gy}$ \\
\hline Spinal cord & max dose $<18 \mathrm{~Gy}$ \\
\hline DVH: dose volume histogram & \\
\hline
\end{tabular}

\section{Plan evaluation and statistics}

The dose to the PTV and OAR was evaluated using DVHs. PTV coverage was compared between VMAT and IMRT using the following parameters:

1. Conformity index $(\mathrm{Cl}, \mathrm{Cl}=\mathrm{VTV} / \mathrm{VPTV})$. Where VTV is the treatment volume enclosed by the prescribed isodose surface, and VPTV is the volume of the PTV. A greater $\mathrm{Cl}$ indicates lower conformity.

2. The homogeneity index $(\mathrm{HI})=\mathrm{D} 5 \% / \mathrm{D} 95 \%$, where $\mathrm{D} 5 \%$ and $\mathrm{D} 95 \%$ are the minimum doses delivered to $5 \%$ and $95 \%$ of the PTV. $\mathrm{HI}$ is a ratio that is used to evaluate the homogeneity of the PTV. The smaller and closer the value of $\mathrm{HI}$ is to 1, the better the homogeneity of the PTV [3].

\section{Statistical analyses}

Non-parametric statistical analyses were performed using the paired, two-tailed Wilcoxon signed-rank test, with a p-value $<0.05$ defined as significant. We compared each patient's IMRT plan with their VMAT plan. 
T-test was used for testing differences in mean coverage of PTV, and to test for differences in the dose to the OAR, among the different planning techniques.

The dosimetric differences among the two treatments for the 20 pts were analysed using the Friedman test. When a significant difference $(p<0.05)$ was found, the difference between two treatments for each effect was further examined by Wilcoxon signed-rank test. All analyses were performed using SPSS software, version 15.0 (SPSS Inc., Chicago, IL).

\section{Results}

The Cl of VMAT plans was superior to those of IMRT $1.0 .7 \pm 0.76$ vs $1.15 \pm 0.14(p<0.013)$.

HI was $1.11 \pm 0.03$ for VMAT and $1.13 \pm 0.04$ for IMRT, with no significant differences among methods ( $p$ $=0.1)$.

IMRT had twice the MUs $(890 \pm 160.68)$ of VMAT $(423.78 \pm 50.65)(p<0.01)$. Although the estimated treatment time delivery was $25 \%$ shorter with VMAT plans $(p<0.046)$ The full data for PTV coverage can be seen in Table 2.

Table 2

Dosimetric parameters for planning target volume coverage

\begin{tabular}{|llll|}
\hline PTV & IMRT & VMAT & $p$ value \\
\hline PTV V95(\%) & 97.9 & 97.6 & $<0.12$ \\
\hline D mean(Gy) & 48.9 & 48.9 & $<016$ \\
\hline Cl & $1.07 \pm 0.76$ & $1.15 \pm 0.14$ & $<0.013$ \\
HI & $1.13 \pm 0.04$ & $1.11 \pm 0.03$ & $<0.1$ \\
\hline $\begin{array}{l}\text { Cl: conformity index; HI: homogeneity index; IMRT: intensity modulated radiotherapy; PTV: planning } \\
\text { treatment volume; VMAT: volumetric modulated arc therapy }\end{array}$ & \\
\hline
\end{tabular}

Stomach maximum dose, normal liver 15 Gy volume, normal liver mean dose were decreased with VMAT planning. However none of the following differed between the plans: V30 Gy of duodenum and small bowel, $10 \mathrm{cc}(\mathrm{Gy})$ of duodenum, mean dose of right kidney and cord max dose. For right lobe tumor VMAT had lower stomach Dmax, normal liver V15, Cl, Spinal Cord Dmax. In left lobe tumors, no difference was found between the plans. Table 3 demonstrates the significant difference in organs at risk sparing between the methods. 
Table 3

Dose-volumetric comparison for OAR of IMRT and VMAT

\begin{tabular}{|llll|}
\hline & IMRT & VMAT & p value \\
\hline Cord 1\%(Gy) & $6.5 \pm 4.7$ & $5.6 \pm 3.3$ & 0.33 \\
\hline Cord dmax(Gy) & $7.9 \pm 5.6$ & $6.1 \pm 3.8$ & 0.1 \\
\hline Small bowel v30Gy(\%) & $0.03 \pm 0.8$ & $0.4 \pm 0.1$ & 0.87 \\
\hline Small bowel D 1\%(Gy) & $5.7 \pm 8.5$ & $5.0 \pm 7.6$ & 0.30 \\
\hline Small bowel dmax(Gy) & $13.3 \pm 18.7$ & $12.6 \pm 17.9$ & 0.30 \\
\hline Stomach v30Gy(\%) & $2.0 \pm 2.1$ & $1.5 \pm 1.9$ & 0.19 \\
\hline Stomach D 1\%(Gy) & $7.7 \pm 5.5$ & $6.8 \pm 5.2$ & 0.16 \\
\hline Stomach dmax(Gy) & $11.3 \pm 7.7$ & $9.5 \pm 7.1$ & 0.03 \\
\hline Duodenum 10 cc(Gy) & $2.2 \pm 3.0$ & $2.7 \pm 3.5$ & 0.23 \\
\hline Duodenum D 1\%(Gy) & $4.2 \pm 7.1$ & $3.8 \pm 6.0$ & 0.68 \\
\hline Duodenum dmax(Gy) & $5.9 \pm 9.8$ & $4.8 \pm 8.9$ & 0.18 \\
\hline Normal liver 5 Gy (\%) & $28.8 \pm 9.1$ & $30.6 \pm 12.4$ & 0.22 \\
\hline Normal liver 7 Gy (\%) & $23.7 \pm 8.0$ & $24.9 \pm 10.6$ & 0.31 \\
\hline Normal liver 15 Gy(cc) & $190 \pm 110$ & $169 \pm 104$ & 0.026 \\
\hline Normal liver mean(Gy) & $5.9 \pm 2.3$ & $5.5 \pm 2.3$ & 0.042 \\
\hline Both kidneys 15 Gy(\%) & $0.6 \pm 2.2$ & $0.4 \pm 1.8$ & 0.23 \\
\hline Rt kidney mean(Gy) & $2.2 \pm 2.7$ & $1.6 \pm 2.5$ & 0.30 \\
\hline OAR: organs at risk; IMRT: intensity modulated radiotherapy; Rt: right; VMAT: volumetric modulated \\
\hline arc therapy & & & \\
\hline
\end{tabular}

\section{Discussion}

In the past decade, radiotherapy has gradually become a treatment option for HCC patients and is currently a standard in several guidelines. Klein and Dawson [5] concluded that SBRT in HCC can have comparable results with other therapies and suggested that it might be offered as a treatment option for early stage HCC patients or patients not eligible to other ablative procedures. SBRT uses up to five fractions utilizing a steep dose fall-off enabling the delivery of high dose to the target volume while limiting the dose to adjacent normal tissue. 
The recent results with SBRT for HCC are very encouraging. Bujold et al. [6] in two sequential trials, showed a 1-year local control of $87 \%$ with a clear association of better outcome with higher SBRT doses, although grade 3 toxicity was observed in one third of the patients. Jang et al. [7] reported that 2-year local control /overall survival (OS ) rates in SBRT treated patients with doses of $>54,45-54$, and $<45$ Gy were $100 / 71,78 / 64$, and $64 \% / 30 \%$, respectively $(p=.009 / p<.001)$. SBRT dose was found to be a statistically significant prognostic factor for OS. A BED 10 of 138.1 (51.1 Gy in 3 fractions) were necessary to achieve $90 \%$ probability of $2 y$ local control. Dose fractionation in this study was $45-54$ Gy in 3 fractions (BED10 of 112.5-151.2 Gy) in Child-Pugh A patients and $30 \mathrm{~Gy}$ in 5 fractions (BED10 of $48 \mathrm{~Gy})$ in Child-Pugh B patients.

The risk of radiation-induced liver disease (RILD) is generally proportional to the mean dose of radiation delivered to normal liver tissue. Sanuki et al. reported 3y survival of $70 \%$ in 185 patients treated with 35 $40 \mathrm{~Gy}$ in 5 fractions with grade 3 RILD of $13 \%$ [8].

In our own experience with SBRT for HCC, we reported favorable outcomes using SBRT as definitive treatment or as a bridge to liver transplantation in pts with early stage inoperable HCC [9]. Median prescribed dose to the tumor was $54 \mathrm{~Gy}$ (range, 30-54Gy) with median overall-survival for the transplanted patients not reached and 23 months for the patients who were not transplanted. Liver explant revealed $81 \%$ response rate ( $27 \%$ complete and 54 partial). Only one patient developed RILD.

This demonstrates that not only is SBRT effective but that it is critical to maximize the therapeutic window in these patients and therefore the importance of the best planning technique.

There is no large-scale trial for assessment the best radiation planning technique for HCC SBRT both for target coverage and normal tissue avoidance.

There are studies which compered VMAT versus IMRT in radiotherapy treatment for hepatocellular carcinoma in conventional fractionated radiotherapy. (50-54Gy using 1.8-2 Gy /day) [10, 11]. VMAT and IMRT was shown to be significantly better $\mathrm{Cl}(0.84 \pm 0.05,0.83 \pm 0.04)$ when compared with 3DCRT $(0.72$ $\pm 0.03, p<0.001)$ in the treatment of $\mathrm{HCC}$ with fractionated regimens $(50 \mathrm{~Gy} / 25$ fractions). More importantly, VMAT showed significant improvement with regard to HI, lower MUs and shorter delivery time compared to IMRT [10].

Park et al. using prescription dose of $54 \mathrm{gy}$ in 30 fractions has reported that the Cl of VMAT $(1.0 \pm 0.008)$ was significantly better than that of IMRT $(1.1 \pm 0.03, p<0.0001)$ [11].

In reviewing studies of SBRT delivery techniques in other tumors, such as lung cancer, pancreatic cancer ,spinal and abdominal metastases, IMRT and VMAT for SBRT and compared those techniques to 3D -CRT with improvement in dose conformality and homogeneity [3]. However, when compared to IMRT dose conformality was greatest with VMAT. Treatment time was markedly quicker with VMAT versus 3DCRT and IMRT. Overall both IMRT and VMAT were able to meet all planning constraints in the studies reviewed; however, treatment efficiency was greatest with VMAT. 
However, to our knowledge, this is the first report comparing IMRT to VMAT in SBRT treated HCC patients. Our data suggests that PTV coverage is more conformal with VMAT planning in all pts. VMAT tended to be more effective in all groups for the sparing of normal liver. For pts with PTV volume $<=46 \mathrm{cc}$ the value of VMAT was better for normal liver V15 and Cl, for PTV volume $>=46 \mathrm{cc}$ no difference was seen. For right lobe tumor VMAT had lower stomach maximum dose, liver $\mathrm{V} 15, \mathrm{Cl}$.

The study has several limitations; It is a small retrospective study, with different prescribed dose to tumor according to Child type of cirrhosis. In addition, the limited sample size makes it difficult to distinguish variable related to the location of disease. In addition, the relationship between PTV size and volumetric sparing efficiency should be further examined to form a conclusive understanding of this relationship.

In conclusion based on our data we suggest further exploration into the use of VMAT strategies for treatment of HCC with SBRT.

\section{Abbreviations}

Cl: conformity index; DVH: dose volume histogram; GTV: gross tumor volume; HCC: hepatocellular carcinoma; HI: homogeneity index; IMRT: intensity modulated radiotherapy; Mus: monitor units; OAR: organs at risk; OS: overall survival; pts: patients; PTV: planning treatment volume; RILD: radiation induced liver disease; SBRT: sereotactic body radiotherapy; VMAT: volumetric modulated arc therapy

\section{Declarations}

\section{Acknowledgements}

Not applicable

\section{Ethics approval and consent to participate}

The study was approved by our local institutional ethics committee, which waived the need for informed consent owing to the retrospective design of the study.

\section{Consent for publication}

Not applicable.

\section{Competing interests}

The authors declare that they have no competing interest

\section{Funding}

This research did not receive any specific grant from funding agencies in the public, commercial, or notfor-profit sectors. 


\section{Authors' contributions}

YK contributed to the study conception and design, data collection, analysis and interpretation of the data, and drafting of the initial manuscript.

YK and AM were responsible for data collection.

MW was responsible for new planning on the CT simulation slides

NG was responsible for the statistical analysis

SS contributed to the study drafting of the initial manuscript,

AP contributed to the study analysis and interpretation of the data, and drafting of the initial manuscript

EF contributed to the study analysis and interpretation of the data, and drafting of the initial manuscript,

BB contributed to the study analysis and interpretation of the data, and drafting of the initial manuscript,

AA contributed to the study analysis and interpretation of the data, and drafting of the initial manuscript,

All authors read and approved the final manuscript

\section{References}

1. Emami B, Lyman J, Brown A, et al. Tolerance of normal tissue to therapeutic irradiation. Int J Radiat Oncol Biol Phys. 1991;21(1):109-22.

2. Russell AH, Clyde C, Wasserman TH, Turner SS, Rotman M. Accelerated hyperfractionated hepatic irradiation in the management of patients with liver metastases: results of the RTOG dose escalating protocol. Int J Radiat Oncol Biol Phys. 1993;27(1):117-23.

3. Sapkaroski D, Osborne C, Knight KA. A review of stereotactic body radiotherapy - is volumetric modulated arc therapy the answer? J Med Radiat Sci. 2015;62(2):142-51.

4. Kuo YC, Chiu YM, Shih WP, et al. Volumetric intensity-modulated Arc (RapidArc) therapy for primary hepatocellular carcinoma: comparison with intensity-modulated radiotherapy and 3-D conformal radiotherapy. Radiat Oncol. 2011;6:76. doi: 10.1186/1748-717X-6-76.

5. Klein J, Dawson LA. Hepatocellular carcinoma radiation therapy: review of evidence and future opportunities. Int J Radiat Oncol Biol Phys. 2013;87(1):22-32.

6. Bujold A, Massev CA, Kim JJ, et al. Sequential phase I and II trials of stereotactic body radiotherapy for locally advanced hepatocellular carcinoma. J Clin Oncol. 2013;31(13):1631-9.

7. Jang WI, Kim MS, Bae SH, et al. High-dose stereotactic body radiotherapy correlates increased local control and overall survival in patients with inoperable hepatocellular carcinoma. Radiat Oncol. 2013;8:250. doi: 10.1186/1748-717X-8-250. 
8. Sanuki N, Takeda A, Oku Y, et al. Stereotactic body radiotherapy for small hepatocellular carcinoma: a retrospective outcome analysis in 185 patients. Acta Oncol. 2014;53(30:399-404.

9. Moore A, Cohen-Naftaly M, Tobar A. Stereotactic body radiation therapy (SBRT) for definitive treatment and as a bridge to liver transplantation in early stage inoperable hepatocellular carcinoma. Radiat Oncol. 2017;12(1):163. doi: 10.1186/s13014-017-0899-4.

10. Chen $D$, Wang $R$, Meng $X$, et al. A comparison of liver protection among 3-D conformal radiotherapy, intensity-modulated radiotherapy and RapidArc for hepatocellular carcinoma. Radiat Oncol. 2014 Feb 6;9:48. doi: 10.1186/1748-717X-9-48.

11. Park JM, Kim K, Chie EK, Choi CH, Ye SJ, Ha SW. RapidArc vs intensity-modulated radiation therapy for hepatocellular carcinoma: a comparative planning study. Br J Radiol. 2012;85(1015):e323-9. 